\title{
Estructura y evidencias de validez de la versión en español del Cuestionario de Valores Psicosociales (QVP-24)
}

\author{
Structure and evidence of validity of the Spanish version of the \\ Psychosocial Values Questionnaire (QVP-24)
}

\author{
Daniela Alonso ${ }^{\mathrm{a}, \mathrm{b}^{*}}$, Silvina Brussino ${ }^{\mathrm{a}, \mathrm{b}}$ \\ anstituto de Investigaciones Psicológicas, Facultad de Psicología, Universidad Nacional de Córdoba, Argentina

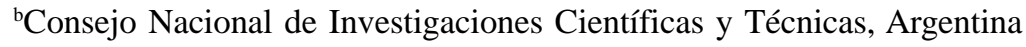

\section{Resumen}

Este artículo presenta evidencia de validez y confiabilidad de la versión en español del Cuestionario de Valores Psicosociales (QVP-24) de Pereira, Camino y Da Costa (2004). Este instrumento se basa en una propuesta teórica que combina perspectivas sociológicas y psicológicas de análisis de los valores. Para su desarrollo se relevaron dos muestras similares de ciudadanos/as de Córdoba (AR) entre 18 y 70 años. Con datos de la primera muestra se condujo un análisis factorial exploratorio; mientras que se utilizaron datos de la segunda para obtener evidencia confirmatoria de la estructura del instrumento. Los resultados arrojaron 4 dimensiones con indicadores de ajuste y consistencia interna adecuados. Además, se obtuvo evidencia de validez concurrente a través de la correlación de estas dimensiones con una escala de autoritarismo del ala de derechas (RWA) (Etchezahar, Cervone, Biglieri, Quattrocchi, \& Prado-Gascó, 2011). Se concluye sobre la validez de este instrumento y su relevancia en el campo de la psicología social y política.

Palabras clave: valores sociales, validez, confiabilidad, propiedades psicométricas.

Para citar este artículo:

Alonso, D., \& Brussino, S. (2018). Estructura y evidencias de validez de la versión en español del Cuestionario de Valores Psicosociales (QVP24). Liberabit, 24(2), 213-230. https://doi.org/10.24265/ liberabit.2018.v24n2.04

\begin{abstract}
This article presents evidence of validity and reliability of the Spanish version of the Psychosocial Values Questionnaire (QVP-24) by Pereira, Camino and Da Costa (2004). This instrument is based on a theoretical approach that combines sociological and psychological perspectives of the social values analysis. Two similar samples of citizens from Córdoba (AR) between 18 and 70 years old were surveyed. With data from the first sample, an exploratory factor analysis was conducted, while data from the second sample was used to obtain confirmatory evidence of the instrument's structure. The results yielded four dimensions, which showed adequate adjustment and internal consistency coefficients. In addition, evidence of concurrent validity was obtained through the correlation of these dimensions with a right wing authoritarianism (RWA) scale (Etchezahar, Cervone, Biglieri, Quattrocchi, \& PradoGascó, 2011). In conclusion, the validity of this instrument and its importance in the field of social and political psychology are confirmed.
\end{abstract}

Keywords: social values, validity, reliability, psychometric properties.

Este es un artículo Open Access bajo la licencia Creative Commons Atribución-NoComercial-CompartirIgual 4.0

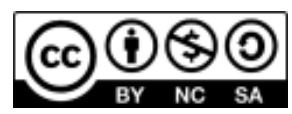




\section{Introducción}

Los valores sociales son dimensiones fundamentales en la organización de los sistemas de creencias de las personas y funcionan como patrones que orientan actitudes, preferencias, juicios y comportamientos sociales (Rokeach, 1973). En consecuencia, se han estudiado ampliamente tanto desde corrientes sociológicas (e.g., Inglehart, 2007; Inglehart, Basañez, \& Moreno, 1998) como desde distintas áreas de la Psicología (e.g., Imhoff \& Brussino, 2013; Schwartz, 1992, 1994, 2005; Sagiv, Roccas, Cieciuch, \& Schwartz, 2017). Las primeras -de las cuales Inglehart $(2000,2007)$ es quizás el representante más destacado- se orientan a analizar los sistemas de valores predominantes en un tiempo y contexto cultural determinado, estudiando sus modificaciones en relación con el desarrollo y transformación de las sociedades. Las segundas -de la cuáles es quizás Schwartz (1992, 1994, 2005) el principal referente- se abocan al análisis de la dimensión individual; es decir, cómo las personas estructuran sus sistemas de valores, qué dimensiones modulan esta estructuración y cómo se vinculan con actitudes y comportamientos. Al mismo tiempo, la mayoría de los desarrollos teórico-empíricos en el área proviene de vertientes europeas, luego aplicadas regionalmente. En este marco, el abordaje psicosocial de los valores que se desarrolla (Pereira, Camino et al., 2004) representa una perspectiva regionalmente situada que conjuga elementos de los enfoques sociológicos y psicológicos, constituyéndose en una herramienta analítica relevante para el abordaje de actitudes y comportamientos sociales y políticos en nuestro contexto.

Las perspectivas psicológicas de análisis de los valores los comprenden como metas motivacionales (individuales) u objetivos deseables, transituacionales, que expresan necesidades básicas de la existencia humana -biológicas y materiales de supervivencia, interacción social y bienestar de los grupos- (Schwartz, 1992, 1994). Así, los valores serían conceptos o creencias que trascienden a situaciones específicas y remiten a fines o comportamientos deseados, guiando el comportamiento (Schwartz, 2003). Además, se ordenan de acuerdo a la importancia relativa que se les adscribe y contribuyen simultáneamente a las actitudes o comportamientos en función de su relevancia para la acción y para el actor. En este marco, Schwartz (1992) postula la existencia de una estructura circular de carácter universal conformada por 10 tipos motivacionales articulados en dos bipolaridades. La primera dimensión opone la apertura al cambio (motivaciones de estimulación y autodirección) a la conservación (seguridad, conformidad y tradición); mientras que la segunda opone la autotrascendencia (universalismo y benevolencia) a la autopromoción (motivaciones de poder y logro); al tiempo que los valores hedonistas responderían tanto a la apertura al cambio como a la autopromoción. En términos generales, el primer eje opone valores que motivan a las personas a seguir sus intereses intelectuales y emocionales, donde se promueve el pensamiento, las experiencias novedosas y la acción independiente a aquellos que preservan el statu quo y la certeza que este proporciona, promoviendo la autorrestricción sumisa y la resistencia al cambio. Por su parte, la segunda bipolaridad opone la preocupación por intereses colectivos a aquellos que enfatizan el éxito y domino personal (Schwartz, 1992).

Por otra parte, las vertientes de análisis sociológico de los valores ubican su origen en las transformaciones culturales que atraviesan las sociedades. Así, Inglehart et al. (1998) señalaban que las prioridades individuales reflejan en gran parte el contexto socioeconómico: el desarrollo económico, político y social generaría cambios graduales en las prioridades de las sociedades, de modo tal que en aquellas que se han resuelto problemas y necesidades básicas (como la seguridad material), se habría ido virando desde preocupaciones materialistas hacia valores posmaterialistas (Inglehart, 1997; Inglehart et al., 1998). Así, los valores materialistas enfatizan en la importancia de la seguridad física y económica y se vinculan con la adhesión a sistemas normativos rígidos y absolutos y a una mayor orientación hacia la autoridad (Inglehart, 
2000; Inglehart et al., 1998). Por su parte, las orientaciones posmaterialistas, propias de contextos de relativa seguridad, desarrollo y estabilidad, expresarían una mayor orientación al cambio social y cultural; priorizando la realización profesional, la política y el bienestar individual, enfatizando así en valores como la expresión, la protección de las libertades y derechos y la tolerancia (Inglehart, 1991, 2000).

A pesar del reconocimiento de su relevancia en la literatura, Pereira, Cardoso y Ribeiro (2005) argumentan que ambas perspectivas resultan reduccionistas: o bien entienden que los valores se originan en motivaciones individuales o bien son productos de los cambios culturales. Consecuentemente, proponen integrar ambos niveles de análisis en un abordaje psicosocial (Barros, Torres, \& Pereira, 2009; Pereira, Camino, \& Da Costa, 2004, 2005), incorporando la dimensión ideológica: los valores sociales constituyen un tipo de conocimiento socialmente estructurado que surge a partir de contenidos ideológicos presentes en la sociedad. Así, una de las condiciones para su desarrollo es la existencia de luchas ideológicas entre grupos sociales en la búsqueda de acceso al poder (Pereira et al., 2005): los valores se anclan en las identidades sociales y posicionamientos ideológicos derivados de ellas (Torres, Pereira, Guimarães, Albernaz, Vieira, \& Barros, 2001). Así, a diferencia de la propuesta de Schwartz (1992), no representarían necesidades individuales, sino identidades ideológicas de grupos sociales (Pereira et al., 2005). Es esta dimensión psicosocial e ideológica la que hace particularmente pertinente la aplicación de este enfoque cuando se analiza la dimensión psicopolítica de las problemáticas sociales y políticas de la actualidad.

Por otra parte, las perspectivas clásicas de los valores han sido objeto de algunas objeciones teóricometodológicas. Una de ellas remite al establecimiento de relaciones de oposición entre las distintas dimensiones. Por ejemplo, la propuesta de Inglehart (1991) ubica a las dimensiones materialistas y posmaterialistas como polos de un continuo, aunque algunos estudios empíricos encuentran que se trata en realidad de dos dimensiones diferentes con algún grado de relación positiva (Pereira et al., 2005). También, como mencionábamos, la tipología de Schwartz (1992) planteaba dos bipolaridades (autopromoción vs. autotrascendencia y conservación vs. apertura al cambio). La perspectiva psicosocial aquí analizada no postula relaciones de oposición entre las dimensiones atendiendo a que, en última instancia, todas refieren a metas deseables, aspecto que se ve respaldado en la evidencia empírica (Pereira et al., 2005).

Respecto de la dimensión metodológica, es la escala de valores de Schwartz (Portrait Values Questionnaire; Schwartz, 2003) la más aplicada globalmente. En el contexto regional, diversos estudios aplicaron tanto su versión completa (e.g., CastroSolano \& Nader, 2006; Delfino \& Muratori, 2011; Delfino \& Zubieta, 2011; Peiró \& Palencia, 2009) como reducida (e.g., Imhoff \& Brussino, 2013; Zubieta, Fernández, \& Sosa, 2012), reportando sus propiedades psicométricas. A pesar de su relevancia para el análisis de aspectos políticos y sociales, en ningún caso se replica la estructura original ni se consiguen indicadores de consistencia adecuados en todas las subescalas (ver también: Hinz, Brähler, Schmidt, \& Albani, 2005; y para un metaanálisis: Steinmetz, Isidor, \& Baeuerle, 2012). Además, se trata de una escala extensa con un planteamiento complejo de sus ítems, lo cual ha generado problemas en su aplicación, por ejemplo, en población sin educación formal (Schwartz, 2003).

Como alternativa a esta forma de evaluación, el QVP-24 resulta de aplicación más sencilla, con buenos indicadores de validez y consistencia, y ha demostrado ser un buen predictor de actitudes y comportamientos sociales y políticos (Barros et al., 2009; Fernandes, Da Costa, Camino, \& Mendoza, 2007; Lins, LimaNunes, \& Camino, 2014; Pereira, Lima, \& Camino, 2001). Más específicamente, evalúa la importancia relativa que las personas le asignan a un conjunto de 24 valores sociales pensando en el desarrollo de una sociedad ideal y los estructura en cuatro dimensiones 
centrales: materialista, posmaterialista, hedonista y religiosa (Lins, Poeschl, De Lima, De Souza, \& Pereira, 2016; Pereira et al., 2001; Pereira, Camino et al., 2004).

Las propiedades psicométricas y la validez convergente de este instrumento fueron presentadas en dos estudios con población universitaria brasilera (e.g., Pereira et al., 2004); además de otra serie de estudios que aplican la escala, pero cuyo objetivo no es presentar evidencia psicométrica del constructo (Pereira, Camino et al., 2004). En el primero de ellos, de carácter descriptivo, se reportaron resultados de análisis de clústeres y escalamiento multidimensional que permitieron replicar la estructura teórica. Además, se estimaron relaciones entre estas y los tipos motivacionales propuestos por Schwartz (1992), encontrando que los valores materialistas se relacionaban positiva y significativamente con valores de poder social; los posmaterialistas, principalmente, con valores de universalismo y benevolencia; los hedonistas, con la estimulación y el hedonismo; y, finalmente, los valores religiosos correlacionaban principalmente con valores de tradición, benevolencia y conformidad. Además, en otro estudio, se reportaron correlaciones entre valores materialistas y la autopromoción, el posmaterialismo y la autotrascendencia, la religiosidad y la conservación, y el hedonismo y la apertura al cambio (Pereira et al., 2005). El segundo estudio psicométrico publicado presenta evidencia de un análisis factorial confirmatorio que también replica la estructura propuesta, mostrando indicadores de ajuste adecuados y cargas factoriales significativas y superiores a .59 en todos los casos. Además, se encontraron relaciones positivas de las dimensiones de los valores entre sí y se reportaron coeficientes de confiabilidad alfa adecuados, superiores a .70 en todos los casos (Pereira, Camino et al., 2004).

Como mencionábamos, desde el punto de vista de su validez predictiva, en distintas investigaciones se ha reportado la pertinencia de esta perspectiva analítica para el abordaje de actitudes y comportamientos psicopolíticos. Por ejemplo, Barros et al. (2009) estudiaron la relación entre los valores psicosociales y el autoritarismo, encontrando que los valores hedonistas se relacionaban positivamente con una mayor tolerancia y negativamente con el autoritarismo; los valores religiosos se asociaban negativamente con la tolerancia y los materialistas lo hacían positivamente con el autoritarismo. En la misma línea, Fernandes et al. (2007) encontraron que los valores materialistas se relacionaban positivamente con la orientación a la dominancia social, mientras que los posmaterialistas lo hacían negativamente. Por su parte, Lins et al. (2014) aplicaron este enfoque para el estudio del prejuicio racial en Brasil, encontrando que los valores psicosociales eran predictores significativos del prejuicio, especialmente cuando se trataba del prejuicio sutil o simbólico. Así, los valores materialistas predecían positivamente el prejuicio, al tiempo que una dimensión de los valores posmaterialistas (justicia social) lo hacía negativamente. En relación con actitudes respecto de dimensiones políticas, Pereira et al. (2001) encontraron que los valores religiosos predecían actitudes negativas hacia la democracia, mientras que los posmaterialistas predecían actitudes de apoyo a esta. Por su parte, en un estudio sobre representaciones sociales sobre los derechos humanos, Pereira, Ribeiro y Cardoso (2004) encontraron que los valores posmaterialistas predecían una visión de que el gobierno debería involucrarse más en la aplicación de los derechos humanos, al tiempo que sucedía lo inverso con los valores materialistas. En términos generales, los valores posmaterialistas han sido consistentemente asociados a actitudes sociales y políticas más progresistas; al tiempo que los valores religiosos y materialistas lo han hecho con posiciones más conservadoras (Brussino, Imhoff, Rabbia, \& PazGarcía, 2013).

Sin embargo, más allá de la relevancia y consistencia de esta evidencia presentada, una de las principales debilidades de este enfoque es que -como mencionábamos- solo ha sido aplicado en 
investigaciones con adolescentes y estudiantes universitarios en contexto brasilero (Lins et al., 2016; Wachelke \& Bueno, 2015). Así, al momento de este estudio no se contaban con versiones en español del Cuestionario de Valores Psicosociales (QVP-24) ni análisis que incluyeran a la población general. Por esta razón, consideramos pertinente no solo realizar una traducción y adaptación de este instrumento al contexto argentino, sino también incluir en nuestra muestra a población de todas las edades y segmentos socioeconómicos que nos permita concluir con mayor confiabilidad acerca de su validez. En función de lo expuesto, este estudio tiene como objetivo analizar las propiedades psicométricas de la versión en español del QVP-24, desarrollado en el contexto latinoamericano y pertinente para un enfoque analítico propio de la Psicología Política (Pereira, Camino et al., 2004).

\section{Método}

\section{Participantes}

Ciudadanos y ciudadanas de Córdoba (Argentina) entre 18 y 70 años de edad. Se relevaron dos muestras con características similares, ambas seleccionadas a través de un muestreo no probabilístico por cuotas de sexo, edad y nivel socioeconómico (en adelante NSE) conformadas en función de los datos de la encuesta anual de hogares urbanos provista por el Instituto Nacional de Estadística y Censos (INDEC) (Muraro, 2012).

Muestra 1: 300 personas, de las cuales el 50\% fueron mujeres y la media de edad de 38.8 años ( $D E$ $=14.15$ ). Respecto del NSE, el $25 \%$ pertenecía a un nivel bajo inferior o marginal; el 26.3\%, a un nivel bajo; el 26.7\%, a un nivel medio bajo; el 16\%, a un nivel medio típico; y el 6\%, a un NSE medio alto o alto.

Muestra 2: 454 personas, de las cuales el 52.6\% eran mujeres y la media de edad de 37.3 años $(D E=$ 14.23). En relación con el NSE el $13.7 \%$ pertenecían a un nivel bajo inferior o marginal; el 31.6\%, a un nivel bajo; el 28.8\%, a un nivel medio bajo; el 18.6\%, a un nivel medio típico; y el 7.3\% restante, a un NSE medio alto $o$ alto.

\section{Instrumentos}

Cuestionario de Valores Psicosociales (QVP-24). Se aplicó una versión en español QVP-24 desarrollado en portugués por Pereira et al. (2004). Esta escala propone 24 valores para evaluar 4 sistemas normativos: 1) materialista (4 ítems; e.g., poder, logro), 2) hedonista (4 ítems; e.g, placer, sensualidad), 3) religioso (4 ítems; e.g., religiosidad, salvación del alma) y 4) posmaterialista (12 ítems; e.g., libertad, igualdad). Las opciones de respuesta en la propuesta original van de 1 (nada importante) a 5 (muy importante) en función de la importancia que atribuye la persona a cada valor, pensando en el desarrollo de una sociedad ideal. Sin embargo, en otros estudios, los mismos autores utilizan un rango de respuestas que van de 1 a 10 (Pereira et al., 2001). Aplicamos la última opción, teniendo en cuenta que es consistente con la mayoría de las propuestas para la evaluación de los valores (e.g., Schwartz, 1992).

Autoritarismo del Ala de Derechas (RWA). Para obtener evidencia de validez concurrente, se incluyó la versión reducida de la escala de RWA adaptada al contexto argentino por Etchezahar et al. (2011). La misma tiene 6 ítems (e.g., Nuestro país necesita un líder poderoso que pueda enfrentar a los extremistas e inmorales que actualmente prevalecen en nuestra sociedad) con formato de respuesta tipo Likert de 5 puntos que conforman una única dimensión que evalúa actitudes generales autoritarias. En nuestra muestra, se replicó la estructura unidimensional con indicadores de ajuste adecuados en un modelo factorial confirmatorio estimado con el método de máxima verosimilitud $\left(\chi^{2}=11.56, \mathrm{gl}=8, p=.172 ; \mathrm{CMIN} / \mathrm{gl}\right.$ $=1.44$; SRMR $=.022$; GFI = .964; CFI = .995; RMSE $=.044$, IC $=.000,-.096)$. La consistencia interna, siguiendo el criterio de Aron y Aron (2001), fue óptima de $\alpha=.88$. En términos de validez del 
constructo, el RWA ha sido asociado en estudios previos con los valores sociales (Barros et al., 2009; Feather \& McKee, 2012) y, más específicamente, esta versión de la escala ha demostrado relaciones positivas y significativas con la centralidad de la religión y las orientaciones religiosas (Etchezahar \& Brussino, 2015).

Se utilizaron variables sociodemográficas para evaluar sexo, edad y nivel educativo, y se construyeron preguntas cerradas de alternativa fija. En el caso del NSE, se tomó un índice que contempla la relación entre cantidad de personas que aportan ingresos y miembros del hogar, la ocupación, nivel educativo y cobertura de obra social del principal sostén del hogar (Comisión de Enlace Institucional, AAM-SAIMO-CEIM, 2006).

\section{Procedimiento}

Para el desarrollo de la versión en español del QVP-24, se aplicó un procedimiento de traducción inversa que implica que cada término (valor) es traducido al español por un experto y, posteriormente, otro experto vuelve a traducirlo al portugués, comparando finalmente la versión original y la retraducida (Werner \& Campbell, como se citó en Vergara \& Balluerka, 2000). Previo a su aplicación en la muestra, se realizó una prueba piloto en un grupo reducido con características similares a la población en estudio para evaluar su comprensión.

El relevamiento de los datos se realizó a través de la aplicación cara a cara de un cuestionario cerrado por parte de entrevistadores/as capacitados/as. La escala que evalúa RWA solo fue aplicada en la muestra 2. Este proceso siguió los lineamientos éticos propuestos por el Consejo Nacional de Investigaciones Científicas y Técnicas (CONICET) en su resolución N. ${ }^{\circ}$ 2857-2006, CONICET: Lineamientos para el comportamiento ético en las Ciencias Sociales y Humanidades. Así, previo a su participación, se explicó a los/as participantes los objetivos del estudio y se solicitó su consentimiento informado, garantizando la confidencialidad y anonimato en el tratamiento de los datos y su utilización con fines exclusivamente académicos. Además, se informó que su participación era voluntaria y podían abandonar el estudio si lo deseaban. La administración de los instrumentos se daba en un único momento, en el espacio elegido por el/la participante y tenía una duración de entre 10 y 15 minutos. Cada muestra fue relevada en un período de 2 meses, con un año de diferencia entre la primera y la segunda.

\section{Análisis de datos}

Los datos fueron procesados utilizando los paquetes estadísticos SPSS 21 (para análisis descriptivos y correlacionales), FACTOR 10.7.1 (para análisis factorial exploratorio) y LISREL 8.8 (para análisis factorial confirmatorio).

En primera instancia, se estimaron los estadísticos descriptivos (media y desviación estándar) para ambas muestras. Además, se calcularon índices de asimetría y curtosis para analizar la normalidad univariada y el índice de Mardia para estimar la normalidad multivariada. También se comprobó la linealidad (por la observación de diagramas matriciales de dispersión) y ausencia de multicolinealidad (correlaciones no superiores a .85) (Pérez \& Medrano, 2010). En segunda instancia, con datos de la muestra 1 se condujeron análisis psicométricos exploratorios. Para conocer la factibilidad de realizar un análisis factorial exploratorio (AFE) se condujo el test de esfericidad de Bartlett -considerando adecuados coeficientes de significación menores a .05- y la prueba de adecuación muestral de Kaiser-Mayer-Olikin (KMO) -considerando adecuados valores iguales o superiores a .70- (Hair, Anderson, Tatham, \& Black, 1999). Una vez comprobados estos supuestos, y teniendo en cuenta que se obtuvieron distribuciones no normales, se estimó el AFE utilizando el método de mínimos cuadrados no ponderados sobre matrices policóricas. Teniendo en cuenta que los valores son dimensiones relacionadas entre sí, se aplicó una rotación oblicua (Promax) (Lloret-Segura, Ferreres-Traver, Hernández-Baeza, \& 
Tomás-Marco, 2014). Como medida de consistencia interna se calculó el alfa de Cronbach, considerando aceptables puntuaciones superiores a .60 y óptimos valores superiores a .70 (Aron \& Aron, 2001). También, se estimó la correlación de cada ítem con la dimensión y el coeficiente alfa si se elimina cada ítem. La interpretación de estos análisis nos permite identificar si hay ítems que no resultan indicadores adecuados de la dimensión. En función de estos resultados -y teniendo en cuenta la estructura teórica de los constructos-, se realizó un análisis factorial confirmatorio (AFC) y pruebas de consistencia interna con datos de la muestra 2. En el primer caso, ateniendo a la no normalidad de la distribución de los datos, se utilizó el método de máxima verosimilitud robusta. Para evaluar el ajuste del modelo, se consignaron los indicadores sugeridos por la literatura (Kline, 2011). Como indicador de ajuste absoluto se estimó el estadístico chi-cuadrado y la discrepancia mínima sobre grados de libertad (CMIN/gl), el índice de bondad del ajuste (GFI) y el residuo cuadrático medio estandarizado (SRMR). Además, como indicador de ajuste incremental, se retomó el índice de ajuste comparativo (CFI); y como indicador de parsimonia, el error cuadrático medio de aproximación (RMSEA). Siguiendo los criterios propuestos por $\mathrm{Hu}$ y Bentler (1995), se consideraron óptimos valores superiores a .95 (y superiores a .90 como aceptables) en CFI y GFI; del RMSEA y SRMR, inferiores a .08; y una CMIN/gl menor a 5, considerando valores menores a 3 como óptimos (Arbuckle, 2003; Kline, 2011). Finalmente, se espera un chi-cuadrado no significativo que evidenciaría que las distancias entre la matriz de covarianzas predicha y los datos pueden ser explicadas por el error de medición. Respecto de la consistencia interna, se volvieron a estimar los coeficientes de confiabilidad alfa de Cronbach y el coeficiente alfa de Cronbach si se elimina cada elemento. También se calcularon sus intervalos de confianza utilizando la prueba de Fischer (DomínguezLara \& Merino-Soto, 2015). Complementariamente, se calculó el coeficiente omega (McDonald, 1999) que emplea las cargas factoriales, brindando mayor estabilidad a los cálculos (Zinbarg, Yovel, Revelle, \& McDonald, 2006).

Una vez confirmada la estructura del constructo, se confeccionaron las subescalas de valores psicosociales a partir de la sumatoria de las puntuaciones de los ítems de cada factor. Como medida de validez concurrente, se calcularon tanto las correlaciones entre estas y el RWA como un modelo de regresión lineal múltiple donde se incluyeron todas las dimensiones de los valores como variables independientes para permitirnos estimar la magnitud de su efecto.

\section{Resultados}

\section{Análisis descriptivos}

En la Tabla 1 se muestran las puntuaciones medias, desviación estándar y coeficientes de asimetría y curtosis de la distribución de los ítems para las dos muestras. Los ítems de la dimensión posmaterialista recogen las puntuaciones medias más altas y los de la dimensión religiosa, las más bajas. Además, muchos ítems arrojan puntuaciones de asimetría y curtosis superiores a lo esperado en función de criterios de normalidad (+/- 1.5) (Pérez \& Medrano, 2010), particularmente en la dimensión posmaterialista. Si bien, con base en las propuestas de Kline (2011) y Pérez, Medrano y Sánchez-Rosas (2013) sostienen que ítems con valores de asimetría no superiores a 3 y de curtosis no superiores a 10 no necesitan ser transformados para su aplicación en análisis multivariados; optamos por aplicar métodos de estimación robustos apropiados para este tipo de distribuciones (Lloret-Segura et al., 2014). Además, en función de la estimación del índice de Mardia, encontramos que, si bien la asimetría multivariada se encontraba dentro de los parámetros aceptables (137.90; $p \leq 1.000$ ), una curtosis multivariada elevada (779.10; $p \leq .001$ ) indicaba que los datos se alejaban significativamente de la normal. 
Tabla 1

Puntuaciones medias, Desviación estándar, asimetría y curtosis de los ítems del Cuestionario de Valores Psicosociales

\begin{tabular}{|c|c|c|c|c|c|c|c|c|}
\hline & \multicolumn{4}{|c|}{ MUESTRA $1(\mathrm{~N}=300)$} & \multicolumn{4}{|c|}{ MUESTRA $2(\mathrm{~N}=454)$} \\
\hline & $M$ & $D E$ & $g_{1}$ & $g_{2}$ & $M$ & $D E$ & $g_{1}$ & $\mathrm{~g}_{2}$ \\
\hline \multicolumn{9}{|l|}{ Posmaterialista } \\
\hline Igualdad & 9.00 & 1.52 & -1.88 & 3.89 & 8.87 & 1.82 & -1.99 & 4.19 \\
\hline Fraternidad & 8.59 & 1.49 & -1.11 & 1.49 & 8.46 & 1.82 & -1.82 & 4.44 \\
\hline Justicia social & 9.16 & 1.42 & -2.43 & 7.89 & 8.94 & 1.91 & -2.33 & 5.58 \\
\hline Libertad & 9.33 & 1.18 & 1.94 & 3.89 & 9.33 & 1.35 & 2.89 & 10.27 \\
\hline Alegría & 8.72 & 1.50 & -1.41 & 2.94 & 8.06 & 1.98 & -1.19 & 1.28 \\
\hline Amor & 9.04 & 1.50 & -2.02 & 5.59 & 8.49 & 1.91 & -1.66 & 3.01 \\
\hline Autorrealización & 8.36 & 1.70 & -1.09 & .78 & 8.10 & 1.94 & -1.14 & 1.27 \\
\hline Competencia & 6.55 & 2.56 & -.53 & -.51 & 5.93 & 2.50 & -.33 & -.57 \\
\hline Comodidad & 7.52 & 2.11 & -.87 & -.39 & 6.88 & 2.18 & -.64 & .12 \\
\hline Dedicación al trabajo & 8.39 & 1.73 & -1.32 & 1.71 & 8.28 & 1.80 & -1.25 & 1.84 \\
\hline Realización profesional & 8.41 & 1.61 & -1.24 & 1.96 & 7.94 & 1.98 & -1.15 & 1.25 \\
\hline Responsabilidad & 9.03 & 1.37 & -1.94 & 4.90 & 8.99 & 1.53 & -2.5 & 8.4 \\
\hline \multicolumn{9}{|l|}{ Materialista } \\
\hline Estatus & 5.78 & 2.56 & -.32 & -.80 & 4.64 & 2.54 & .03 & -1.05 \\
\hline Riqueza & 6.65 & 2.49 & -.52 & -.36 & 5.25 & 2.67 & -.17 & -.98 \\
\hline Autoridad & 7.37 & 2.24 & -.98 & .59 & 6.71 & 2.45 & -.69 & -.17 \\
\hline Lucro & 5.71 & 2.58 & -.25 & -.64 & 4.73 & 2.66 & .08 & -1.05 \\
\hline \multicolumn{9}{|l|}{ Religiosa } \\
\hline Salvación del alma & 5.54 & 3.32 & -.13 & -1.41 & 4.09 & 3.39 & .60 & -1.21 \\
\hline Obediencia a la ley de Dios & 5.99 & 3.27 & -.32 & -1.27 & 4.18 & 3.30 & .53 & -1.21 \\
\hline Religiosidad & 5.81 & 3.07 & -.33 & -1.17 & 4.04 & 3.10 & .52 & -1.13 \\
\hline Temor de Dios & 4.91 & 3.50 & .19 & -1.57 & 3.26 & 3.10 & 1.10 & -.25 \\
\hline \multicolumn{9}{|l|}{ Hedonista } \\
\hline Placer & 8.00 & 2.00 & -1.29 & 1.81 & 6.98 & 2.51 & -.89 & .07 \\
\hline Sexualidad & 7.77 & 2.20 & -1.18 & 1.21 & 6.95 & 2.59 & -.84 & -.12 \\
\hline Sensualidad & 6.68 & 2.30 & -.47 & -.29 & 5.45 & 2.83 & -.18 & -1.09 \\
\hline Una vida excitante & 6.72 & 2.41 & -.49 & -.43 & 5.50 & 2.62 & -.21 & -.88 \\
\hline
\end{tabular}

Nota: $M=$ Media; $D E=$ Deviación estándar; $\mathrm{g}_{1}=$ asimetría; $\mathrm{g}_{2}=$ curtosis.

\section{Análisis factorial exploratorio}

Los análisis de factibilidad de realizar un análisis factorial arrojaron resultados satisfactorios $(\mathrm{KMO}=$ .82; $p \leq .001)$. En función de ello, se procedió a la estimación del AFE con el método de mínimos cuadrados no ponderados sobre matrices policóricas. Se identificaron 4 dimensiones que explicaron el $62.6 \%$ de la varianza. Sin embargo, los valores comodidad y competencia, teóricamente posmaterialistas, cargaron sobre la dimensión materialista. Complementariamente, los análisis descriptivos de la relación de los ítems con la dimensión confirman que estos tienen una correlación baja con la dimensión a la que teóricamente pertenecen y su exclusión no 
afecta significativamente la confiabilidad de la escala (Tabla 2). Más aún, si se remueven ambos ítems, esta se incrementa a $\alpha=.82$. Para el resto de las dimensiones, la consistencia interna también fue óptima ( $\alpha=.80$ a $\alpha=.92)$.

Tabla 2

Correlación ítem-escala corregida y coeficiente alfa si se elimina el ítem para las dimensiones del Cuestionario de Valores Psicosociales

\begin{tabular}{lll}
\hline & $\mathrm{r}_{\text {itc }}$ & Alfa si se elimina ítem \\
\hline Posmaterialista $(\alpha=.79)$ & & \\
Igualdad & .56 & .77 \\
Fraternidad & .56 & .77 \\
Justicia social & .46 & .78 \\
Libertad & .36 & .79 \\
Alegría & .48 & .78 \\
Amor & .48 & .78 \\
Autorrealización & .42 & .78 \\
Competencia & .29 & .81 \\
Comodidad & .38 & .79 \\
Dedicación al trabajo & .49 & .78 \\
Realización profesional & .59 & .77 \\
Responsabilidad & .45 & .78 \\
Materialista ( $\alpha=.81)$ & & \\
Estatus & .72 & .72 \\
Riqueza & .69 & .73 \\
Autoridad & .55 & .80 \\
Lucro & .56 & .79 \\
Religiosa $(\alpha=.92)$ & & \\
Salvación del alma & .64 & .75 \\
Obediencia a la ley de Dios & .66 & .74 \\
Religiosidad & .57 & .78 \\
Temor de Dios & .61 & .76 \\
Hedonista ( $\alpha=.80)$ & & .73 \\
Placer & .68 & .75 \\
Sexualidad & .63 & \\
Sensualidad & .52 & \\
Una vida excitante & & \\
\hline
\end{tabular}

Nota: $\mathrm{r}_{\mathrm{itc}}=$ correlación ítem escala corregida. 
En función de esta evidencia y en comunicación con los autores de la escala original, se decide quitar los dos ítems problemáticos de la dimensión posmaterialista. Así, se repitió el procedimiento del AFE: excluyéndolos. Se obtuvo un modelo factorial de 4 dimensiones que explicaron el $64.77 \%$ de la varianza en la muestra $1(\mathrm{KMO}=.81 ; p \leq .001)$. En la Tabla 3 se exponen las cargas factoriales de cada ítem sobre la dimensión.

Tabla 3

Análisis factorial exploratorio del Cuestionario de Valores Psicosociales. Método de mínimos cuadrados no ponderados con rotación Promax

\begin{tabular}{|c|c|c|c|c|}
\hline & \multicolumn{4}{|c|}{ Componente } \\
\hline & Posmaterialista & Materialista & Religiosa & Hedonista \\
\hline Igualdad & .83 & & & \\
\hline Fraternidad & .70 & & & \\
\hline Justicia social & .73 & & & \\
\hline Libertad & .60 & & & \\
\hline Alegría & .50 & .39 & & \\
\hline Amor & .55 & & & \\
\hline Autorrealización & .47 & .41 & & \\
\hline Dedicación al trabajo & .50 & .39 & & \\
\hline Realización profesional & .60 & & & \\
\hline Responsabilidad & .66 & & & \\
\hline Estatus & & .84 & & \\
\hline Riqueza & .35 & .64 & & \\
\hline Autoridad & & .68 & & \\
\hline Lucro & & .54 & & \\
\hline Salvación del alma & & & .81 & \\
\hline Obediencia a la ley de Dios & & & .85 & \\
\hline Religiosidad & & & .98 & \\
\hline Temor de Dios & & & .97 & \\
\hline Placer & & & & .68 \\
\hline Sexualidad & & .35 & & .61 \\
\hline Sensualidad & & & & .75 \\
\hline Una vida excitante & & & & .78 \\
\hline
\end{tabular}

Nota: Se suprimen las cargas factoriales menores a .30

\section{Análisis factorial confirmatorio}

Replicando la estructura del análisis exploratorio, se condujo un AFC con datos de la muestra 2. Para ello, se utilizó el método de máxima verosimilitud robusta. En términos generales, el modelo propuesto tuvo un ajuste adecuado $\left(\chi^{2}=533.04, \mathrm{gl}=197, p \leq\right.$ $.001 ; \mathrm{CMIN} / \mathrm{gl}=2.70 ; \mathrm{SRMR}=.08 ; \mathrm{GFI}=.88 ; \mathrm{CFI}$
$=.96 ;$ RMSEA $=.061$, IC 90\% (.055 - .068). Si bien el estadístico chi-cuadrado dio un resultado significativo que es no esperado, el CMIN/gl resultó dentro de los límites aceptables, aportando evidencia sobre el ajuste global del modelo. En esta línea, la literatura sugiere que, debido al tamaño de la muestra, no sería acertado rechazar un modelo sobre la única base del estadístico 
chi-cuadrado, siendo más relevante analizar de modo integral su grado de ajuste (Batista-Foguet, Coenders, \& Alonso, 2004). Por su parte, el índice global del ajuste (GFI) presentó un coeficiente apenas por debajo de lo esperado (.88). Atendiendo a este resultado, se estimó una medida de ajuste complementario calculando el índice de ajuste no normado (NNFI) de Tucker y Lewis, que es independiente del tamaño muestral y contempla la parsimonia del modelo, además de su ajuste. Este arrojó un coeficiente de .95 que señala un buen ajuste (Batista-Foguet et al., 2004). Al mismo tiempo, el resto de los indicadores brindó resultados adecuados y, además, todas las cargas factoriales fueron superiores a $.40 \mathrm{y}$ estadísticamente significativas (Tabla 4).
A continuación, se calculó el coeficiente alfa de Cronbach para cada dimensión como medida de consistencia interna y, complementariamente, se estimó el coeficiente omega según la propuesta de McDonald (1999). Los resultados fueron óptimos en todos los casos. Para los valores posmaterialistas fue de $\alpha=.84$ (IC $95 \%=.81-.87$ ) y de $\omega=.83$; para valores religiosos, de $\alpha=.93$ (IC 95\% = .91 - .94) y de $\omega=$ .97 ; para valores materialistas, de $\alpha=.80$ (IC $95 \%=$ $.76-.84)$ y de $\omega=.84$; y para valores hedonistas, de $\alpha=.82$ (IC $95 \%=.78-.85$ ) y de $\omega=.90$. Finalmente, las covarianzas entre las dimensiones son consistentes con los hallazgos de la literatura previa (Pereira et al., 2004) que reportan relaciones positivas entre ellas: posmaterialista versus materialista $(p=.20 ; p<.001)$;

\section{Tabla 4}

Estructura del Cuestionario de Valores Psicosociales. Análisis factorial confirmatorio

\begin{tabular}{|c|c|c|c|c|}
\hline \multirow[b]{2}{*}{ Ítems } & \multicolumn{4}{|c|}{ Dimensión } \\
\hline & Posmaterialista & Materialista & Religiosa & Hedonista \\
\hline Igualdad & $.44^{*}$ & & & \\
\hline Fraternidad & $.55 *$ & & & \\
\hline Justicia social & $.54 *$ & & & \\
\hline Libertad & $.57 *$ & & & \\
\hline Alegría & $.49 *$ & & & \\
\hline Amor & $.51 *$ & & & \\
\hline Autorrealización & $.68^{*}$ & & & \\
\hline Dedicación al trabajo & $.68^{*}$ & & & \\
\hline Realización profesional & $.62 *$ & & & \\
\hline Responsabilidad & $.69 *$ & & & \\
\hline Estatus & & $.74^{*}$ & & \\
\hline Riqueza & & $.83 *$ & & \\
\hline Autoridad & & $.74^{*}$ & & \\
\hline Lucro & & $.69 *$ & & \\
\hline Salvación del alma & & & $.98 *$ & \\
\hline Obediencia a la ley de Dios & & & $.96^{*}$ & \\
\hline Religiosidad & & & $.90 *$ & \\
\hline Temor de Dios & & & $.90 *$ & \\
\hline Placer & & & & $.85 *$ \\
\hline Sexualidad & & & & $.71^{*}$ \\
\hline Sensualidad & & & & $.80 *$ \\
\hline Una vida excitante & & & & $.94 *$ \\
\hline
\end{tabular}


posmaterialista versus religiosa $(p=.19 ; p<.001)$; posmaterialista versus hedonista $(p=.23 ; p<.001)$; materialista versus religiosa $(p=.42 ; p<.001)$; materialista versus hedonista $(p=.35 ; p<.001)$; y religiosa versus hedonista $(p=.12 ; p<.05)$. Las dimensiones más asociadas entre sí son las que remiten a valores religiosos y materialistas, al igual que los valores hedonistas con los materialistas. Las menos relacionadas son las que remiten a valores religiosos respecto a los posmaterialistas y hedonistas.

\section{Validez concurrente}

Para aportar evidencia de validez concurrente, se estimaron las correlaciones de estas dimensiones con el autoritarismo del ala de derechas (RWA). Como mencionábamos, algunas dimensiones de los valores se asocian consistentemente a actitudes más progresistas (los valores posmaterialistas en la perspectiva psicosocial y sociológica, y la autotrascendencia y apertura al cambio en la perspectiva psicológica), mientras que otras lo hacen con actitudes conservadoras (las dimensiones materialista y religiosa, y las de conservación y autodirección). Más específicamente, no solo desde la perspectiva psicosocial se ha comprobado la relación entre los valores sociales y el RWA (Barros et al., 2009), sino que también se han reportado resultados similares desde perspectivas psicológicas. Así, Feather y McKee (2012), en una muestra de estudiantes universitarios australianos, encontraron que la autodirección y el universalismo se relacionaban negativamente con el RWA, mientras que la tradición, conformidad y seguridad lo hacían positivamente. Resultados similares reportó otro estudio con una muestra alemana más amplia (Cohrs, Moschner, Maes, \& Kielmann, 2005). Por su parte, desde otra perspectiva de los valores, Heaven y sus colaboradores (Heaven \& Connors, 2001; Heaven, Organ, Supavadeeprasit, \& Leeson, 2006) encontraron relaciones positivas y significativas entre el RWA y valores vinculados a la fuerza y el orden, valores religiosos, de seguridad (relaciones seguras y satisfactorias) y conservación (modos apropiados de vestir y comportarse); al tiempo que no encontraban relaciones entre el RWA y valores de armonía e igualdad.

Por nuestra parte, estimamos la correlación y regresión múltiple de los valores sobre el RWA como medida de validez concurrente (Tabla 5).

Reportamos relaciones bivariadas positivas y fuertes entre el RWA y la dimensión materialista (.44; $p \leq .001)$ y religiosa $(.58 ; p \leq .001)$ (típicamente asociados a orientaciones conservadoras). Por su parte, la relación con las dimensiones de los valores que la literatura vincula a orientaciones ideológicas más liberales con el autoritarismo fue más débil: para el posmaterialismo resultó no significativa (.04) y para el hedonismo, la relación -aunque significativa- fue débil y en el sentido opuesto al esperado (.11; $p \leq$ .05). Respecto de su aporte conjunto a la explicación

Tabla 5

Estimación de validez concurrente. Modelo de regresión lineal múltiple

\begin{tabular}{lcc}
\hline & $\beta$ & $r$ \\
\hline Valores materialistas & $-27 *$ & $.44 * * *$ \\
Valores religiosos & $.50 * * *$ & $.58 * * *$ \\
Valores posmaterialistas & $-.10 * * *$ & .02 \\
Valores hedonistas & -.02 & $.11^{*}$ \\
$\mathrm{R}$ & .63 & \\
$R^{2}$ & .40 & \\
\hline
\end{tabular}

Nota: ${ }^{*} p \leq .05 ;{ }^{* * *} p \leq .001$ 
del RWA, los valores permiten explicar un $40 \%$ de la varianza, siendo las dimensiones materialistas y religiosas las que tienen un efecto mayor y positivo. Cuando se consideran en conjunto, las dimensiones liberales de los valores (posmaterialismo y hedonismo) predicen de modo negativo el RWA tal como era esperable teóricamente, aunque su efecto sigue siendo débil y, para el hedonismo, no significativo.

\section{Discusión}

Los resultados expuestos nos permitieron aportar evidencia sobre la confiabilidad y validez de la versión en español del Cuestionario de Valores Psicosociales (QVP-24; Pereira, Camino et al., 2004). Hasta el momento de este estudio no se tiene conocimiento de la aplicación de este enfoque en contextos diferentes al desarrollo de la escala, tratándose esta de la primera aplicación al español y, también por primera vez, con una población no universitaria. Así, el principal aporte que creemos realiza este estudio es proveer evidencia de validez de un instrumento que -a diferencia de los enfoques psicológicos provenientes principalmente de la tradición de Schwartz (1992, 2003)-, ha demostrado una estructura consistente y estable en distintos contextos (Barros et al., 2009; Pereira et al., 2001; Pereira, Camino et al., 2004, 2005; Pereira, Cardoso et al., 2005; Pereira, Ribeiro et al., 2004). Además, se trata de una perspectiva particularmente relevante para el análisis de las dimensiones normativas subyacentes a actitudes y comportamientos sociales y políticos; reconociendo el carácter psicosocial de este constructo, recuperando la dimensión ideológica que lo atraviesa (Pereira, Cardoso et al., 2005). En consecuencia, creemos que resulta un aporte valioso para la Psicología Social y Política latinoamericana, proveyendo la validación en español de un instrumento de aplicación sencilla que aborda empíricamente una variable de interés para el área; y lo hace desde una perspectiva psicosocial y situada, surgida de sólidas líneas de trabajo regionales.

Desde el punto de vista técnico, la metodología aplicada -que incluyó una fase exploratoria y una fase confirmatoria con muestras diferentes- robustece la fiabilidad de los resultados. Así, el análisis factorial exploratorio nos permitió identificar cuatro dimensiones relacionadas, coincidentes con la propuesta de estudios psicométricos previos de la versión en portugués (Pereira et al., 2001; Pereira, Camino et al., 2004); aunque se debió eliminar dos ítems de la dimensión posmaterialista (conformidad y comodidad) que no cargaban sobre el factor al que teóricamente correspondían. Como señalamos oportunamente, esta decisión se tomó siguiendo un criterio estadístico, pero contemplando también su correspondencia con la definición conceptual de las dimensiones y en comunicación personal con los autores de la versión original. Al mismo tiempo, no afectó su capacidad explicativa y, en todos los casos, se obtuvieron indicadores de consistencia interna óptimos. Por otra parte, el hecho de contar con una segunda muestra para estimar el AFC permitió comprobar la adecuación de estas modificaciones. En este último caso, con métodos estadísticos equivalentes a la literatura antecedente (Pereira et al., 2004), se arribaron a resultados semejantes; replicando una estructura de 4 dimensiones (valores materialistas, religiosos, hedonistas y posmaterialistas) que mantienen relaciones de adyacencia entre sí tal como plantea este enfoque (e.g., Pereira, Camino et al., 2005) y en contradicción con perspectivas psicologicistas como la de Schwartz (1992).

De modo más específico, las dimensiones materialista y religiosa -asociadas en la literatura a actitudes y comportamientos políticos conservadoresfueron las que obtuvieron mejores indicadores de consistencia interna y validez concurrente; siendo las que realizaron un aporte explicativo mayor al RWA. Por su parte, la relación de las dimensiones de los valores que la literatura vincula a orientaciones ideológicas más liberales (posmaterialismo y hedonismo) con el autoritarismo fue más débil, lo cual replica lo reportado en la literatura citada (e.g., Heaven \& Connors, 2001; Heaven et al., 2006). La dimensión hedonista de los valores podría ser objeto de un análisis posterior dado que, si bien la literatura 
antecedente la relacionaba con orientaciones ideológicas progresistas, en nuestro caso tuvo correlaciones más elevadas con la dimensión materialista y, en términos bivariados, se asoció positiva, aunque débilmente, con un mayor RWA. Así, los valores hedonistas mostraron una tendencia similar a los típicamente conservadores; lo cual podría explicarse en función de que esta, al igual que el materialismo, tiene un carácter individualista. En la misma línea, Fernandes, Da Costa, Camino y Mendoza (2006) habían encontrado una superposición parcial entre los elementos de la dimensión hedonista y materialista de los valores. Para el caso de la dimensión posmaterialista, esta generaba una alta adhesión en toda la muestra (las puntuaciones medias eran superiores a 7.5 en todos los ítems), lo cual podría explicar que sea menos potente en términos de su capacidad discriminante y predictiva de actitudes y comportamientos políticos tales como el RWA en nuestro estudio u otras dimensiones como las actitudes favorables hacia la democracia, la tolerancia política o el autoposicionamiento ideológico (e.g., Alonso, 2018). Además, datos de estudios previos con población similar a la nuestra señalaron que las orientaciones ideológicas y normativas asociadas a actitudes y comportamientos progresistas manifiestan una menor consistencia y estabilidad (e.g., Brussino, Paz-García, Rabbia, \& Imhoff, 2011). Una explicación posible para ello radica en la mayor rigidez y cierre cognitivo asociada a orientaciones conservadoras (Calogero, Bardi, \& Sutton, 2009).

En función de estos hallazgos, consideramos relevante profundizar en el estudio de las dimensiones hedonista y posmaterialista de los valores y su relación con orientaciones ideológicas y políticas progresistas; así como con variables asociadas a la apertura o rigidez cognitiva. Al mismo tiempo, debido a la alta adhesión a estos valores en general, consideramos pertinente evaluar la intervención de la deseabilidad social en estas respuestas.
Finalmente, consideramos pertinente señalar una limitación metodológica de este estudio que radica en el tipo de muestreo. Si bien incluir una muestra cuotificada de población de todas las edades y niveles educativos y socioeconómicos representa un avance respecto de estudios previos (e.g., Pereira et al., 2004), el hecho de no contar con muestras probabilísticas no permite la generalización de los resultados a la población general. Sobre este aspecto podría también avanzarse en estudios posteriores.

\section{Conflicto de intereses}

Las autoras dejan constancia de que no existe ningún conflicto de intereses (morales, económicos, laborales o de investigación) que pueda haber interferido con el desarrollo de este trabajo.

\section{Responsabilidad ética}

Para el trabajo con participantes humanos/as se siguieron los lineamientos propuestos por el Consejo Nacional de Investigaciones Científicas y Técnicas (CONICET) en su resolución N. ${ }^{\circ}$ 2857-2006, CONICET: Lineamientos para el comportamiento ético en las Ciencias Sociales y Humanidades. De este modo, todas las personas brindaron su consentimiento informado. Además, cada participante potencial fue previamente informado sobre los propósitos, métodos, beneficios anticipados y riesgos potenciales de su participación. También, se les informó que podían abstenerse de participar y que podían retirar su consentimiento en cualquier momento. Además, se garantizó el anonimato en el tratamiento y publicación de los datos; los cuales se utilizarán únicamente con fines académicos. Esta información está disponible en la sección de «Procedimiento» del manuscrito. 


\section{Referencias}

Alonso, D. (2018). Análisis Multidimensional de Legitimidad Política (Tesis doctoral). Universidad Nacional de Córdoba, Argentina.

Arbuckle, J. L. (2003). Amos user's guide. Chicago: Small Waters.

Aron, A., \& Aron, E. (2001). Estadística para Psicología. Buenos Aires: Pearson Educación.

Barros, T. S., Torres, A. R. R., \& Pereira, C. (2009). Autoritarismo e adesão a sistemas de valores psicossociais. Psico-USF, 14(1), 47-57.

Batista-Foguet, J. M., Coenders, G., \& Alonso, J. (2004). Análisis factorial confirmatorio. Su utilidad en la validación de cuestionarios relacionados con la salud. Medicina clínica, 122(1), 21-27.

Brussino, S. A., Imhoff, D. S., Rabbia, H. H., \& PazGarcía, A. P. (2013). Ideología política en torno a issues y valores sociales: un estudio correlacional en ciudadanos de Córdoba/Argentina. América Latina Hoy, 65, 161-182. doi: 10.14201/alh201365161182

Brussino, S. A., Paz-García, A. P., Rabbia, H. H., \& Imhoff, D. (2011). Ideología política y prácticas mediatizadas de consumo informativo. Revista Debates, 5(2), 13-40. doi: 10.22456/1982-5269. 22678

Calogero, R. M., Bardi, A., \& Sutton, R. M. (2009). A need basis for values: Associations between the need for cognitive closure and value priorities. Personality and Individual Differences, 46(2), 154-159. doi: 10. 1016/j.paid.2008.09.019

Castro-Solano, A., \& Nader, M. (2006). La evaluación de los valores humanos con el Portrait Values Questionnaire de Schwartz. Interdisciplinaria, 23(2), 155-174. Recuperado de http://www.redalyc.org/ articulo.oa?id=18023202

Cohrs, J. C., Moschner, B., Maes, J., \& Kielmann, S. (2005). The motivational bases of right-wing authoritarianism and social dominance orientation: Relations to values and attitudes in the aftermath of September 11, 2001. Personality and Social Psychology Bulletin, 31(10), 1425-1434. doi: 10.1 $177 / 0146167205275614$
Comisión de Enlace Institucional, AAM-SAIMO-CEIM (2006). Nivel Socio Económico 2006. Recuperado de http:/www.saimo.org.ar/archivos/observatorio-social/ El-NSE-en-la-Argentina-2015-Estratificacion-yVariables.pdf

Delfino, G. I., \& Muratori, M. (2011, noviembre). Análisis factorial confirmatorio de la escala de valores de Schwartz. Trabajo presentado en el 3er. Congreso Internacional de Investigación, La Plata, Argentina.

Delfino, G. I., \& Zubieta, E. M. (2011). Valores y política. Análisis del perfil axiológico de los estudiantes universitarios de la ciudad de Buenos Aires (República Argentina). Interdisciplinaria, 28(1), 93114. Recuperado de http://www.redalyc.org/ articulo.oa?id=18022327006

Domínguez-Lara, S., \& Merino-Soto, C. (2015). ¿Por qué es importante reportar los intervalos de confianza del coeficiente alfa de Cronbach? Revista Latinoamericana de Ciencias Sociales, niñez y Juventud, 13(2), 1326-1328. Recuperado de http:// www.redalyc.org/articulo.oa?id=77340728053

Etchezahar, E., \& Brussino, S. (2015). Análisis de las relaciones entre las dimensiones del autoritarismo, la centralidad de la religión y las orientaciones religiosas: diferencias en el análisis lineal y no lineal de sus relaciones. Actualidades en Psicología, 29(118), 73-81. doi: 10.15517/ap.v29i118.18210

Etchezahar, E., Cervone, N., Biglieri, J., Quattrocchi, P., \& Prado-Gascó, V. (2011). Adaptación y validación de la versión reducida de la escala de autoritarismo de derechas (RWA) al contexto argentino. Anuario de investigaciones, 18, 237-242. Recuperado de http:// www.redalyc.org/articulo.oa?id=369139947026

Feather, N. T., \& McKee, I. R. (2012). Values, right wing authoritarianism, social dominance orientation, and ambivalent attitudes toward women. Journal of Applied Social Psychology, 42(10), 2479-2504. doi: 10.1111/j.1559-1816.2012.00950.x

Fernandes, S. C., Da Costa, J., Camino, L., \& Mendoza, R. (2006). Valores psicossociais e participação política de estudantes universitários de uma cidade do nordeste brasileiro. Psicologia Política, 6(11), 35-64. 
Fernandes, S., Da Costa, J., Camino, L., \& Mendoza, R. (2007). Valores psicossociais e orientação à dominância social: Um estudo acerca do preconceito. Psicologia: reflexão e crítica, 20(3), 490-498. doi: 10.1590/S0102-79722007000300017

Hair, J. F., Anderson, R. E., Tatham, R. L., \& Black, W. C. (1999). Análisis multivariante (Vol. 491). Madrid: Prentice Hall.

Heaven, P. C., \& Connors, J. R. (2001). A note on the value correlates of social dominance orientation and right-wing authoritarianism. Personality and Individual Differences, 31(6), 925-930. doi: 10.10 16/S0191-8869(00)00194-X

Heaven, P. C., Organ, L. A., Supavadeeprasit, S., \& Leeson, P. (2006). War and prejudice: A study of social values, right-wing authoritarianism, and social dominance orientation. Personality and Individual Differences, 40(3), 599-608. doi: 10.1016/j.paid. 2005.08.005

Hinz, A., Brähler, E., Schmidt, P., \& Albani, C. (2005). Investigating the circumplex structure of the Portrait Values Questionnaire (PVQ). Journal of Individual Differences, 26(4), 185-193. doi: 10.1027/16140001.26.4.185

Hu, L., \& Bentler, P. M. (1995). Evaluating model fit. In R. H. Hoyley (Ed.). Structural equation modeling: concepts, issues and applications (pp. 76-99). Thousand Oaks, California: Sage Publications, Inc.

Imhoff, D., \& Brussino, S. (2013). Estudio exploratorio de las características psicométricas del Portrait Values Questionnaire en el contexto de CórdobaArgentina. Revista colombiana de psicología, 22(1), 135-149. Recuperado de http://www.redalyc.org/ articulo.oa? $\mathrm{id}=80428081010$

Inglehart, R. (1991). El cambio cultural en las sociedades industriales avanzadas. Madrid, España: Siglo XXI.

Inglehart, R. (1997). Modernization and posmodernization: cultural, economic, and political change in 43 societies. Princeton: Princeton University Press.
Inglehart, R. (2000). Globalization and postmodern values. The Washington Quarterly, 23(1), 215-228. doi: 10.1162/016366000560665

Inglehart, R. (2007). Mapping Global Values. In Y. Esmer \& T. Pettersson (Eds.), Measuring and Mapping Cultures: 25 Years of Comparative Value Surveys (pp. 115-136). Leiden: Brill.

Inglehart, R., Basañez, M., \& Moreno, A. (1998). Human values and beliefs: a cross-cultural sourcebook: political, religious, sexual, and economic norms in 43 societies. Ann Harbor, Michigan: University of Michigan Press.

Kline, R. B. (2011). Principles and practice of structural equation modeling (3rd. ed.). New York: Guilford Press.

Lins, S., Lima-Nunes, A. V., \& Camino, L. (2014). O papel dos valores sociais e variáveis psicossociais no preconceito racial brasileiro. Psicologia \& Sociedade, 26(1), 95-105.

Lins, S., Poeschl, G., De Lima, T. J. S., De Souza, L. E. C., \& Pereira, C. R. (2016). Adaptação e Validação do Questionário de Valores Psicossociais (QVP-24) ao Contexto de Adolescentes brasileiros e portugueses. Psicologia: Reflexão e Crítica / Psychology: Research and Review, 29(12), 1-10. doi: 10.1186/ s41155-016-0013-0

Lloret-Segura, S., Ferreres-Traver, A., Hernández-Baeza, A., \& Tomás-Marco, I. (2014). El análisis factorial exploratorio de los ítems: una guía práctica, revisada y actualizada. Anales de Psicología, 30(3), 11511169. doi: 10.6018/analesps.30.3.199361

McDonald, R. P. (1999). Test theory: A unified treatment. Mahwah, New Jersey: Erlbaum.

Muraro, O. (2012). El NSE en la encuesta anual de hogares urbanos (INDEC). Sociedad Argentina de Investigadores de Marketing y Opinión. Recuperado de http://www.saimo.org.ar/archivos/observatoriosocial/NSE-en-la-EAHU.pdf

Peiró, S., \& Palencia, E. (2009). Adaptación transcultural del cuestionario The Portrait Values Questionnaire (PVQ) IV versión basada en el modelo de «HRQOL instruments». Educación en valores, 1(11), 9-17. 
Pereira, C., Camino, L., \& Da Costa, J. (2004). Análise fatorial confirmatória do Questionário de Valores Psicossociais-QVP24. Estudos de psicologia, 9(3), 505-512. Recuperado de http://www.scielo.br/pdf/ epsic/v9n3/a13v09n3.pdf

Pereira, C., Camino, L., \& Da Costa, J. (2005). Um estudo sobre a integração dos níveis de análise dos sistemas de valores. Psicologia: reflexão $e$ crítica, 18(1), 16-25. Recuperado de http://www.reda lyc.org/artic ulo.oa?id=18818104

Pereira, C., Cardoso, S. J., \& Ribeiro, A. R. C. (2005). Teste empírico de um modelo sobre as relações entre os sistemas de valores e as atitudes. Psicologia, 19(12), 227-250.

Pereira, C., Lima, M. E., \& Camino, L. (2001). Sistemas de valores e atitudes democráticas de estudantes universitários de João Pessoa. Psicologia: Reflexão e Crítica, 14(1), 177-190. Recuperado de http://www. scielo.br/pdf/prc/v14n1/5217.pdf

Pereira, C., Ribeiro, A. R. C., \& Cardoso, S. J. (2004). Envolvimento nos direitos humanos e sistemas de valores. Psicologia em estudo, 9(1), 55-65. doi: 10.1 590/S1413-73722004000100008

Pérez, E. R., \& Medrano, L. A. (2010). Análisis factorial exploratorio: bases conceptuales y metodológicas. Revista Argentina de Ciencias del Comportamiento (RACC), 2(1), 58-66.

Pérez, E. R., Medrano, L. A., \& Sánchez-Rosas, J. (2013). El Path Analysis: conceptos básicos y ejemplos de aplicación. Revista Argentina de Ciencias del Comportamiento (RACC), 5(1), 52-66. Recuperado de http://www.redalyc.org/articulo.oa?id=333427385008

Rokeach, M. (1973). The nature of human values. Michigan: Free press.

Sagiv, L., Roccas, S., Cieciuch, J., \& Schwartz, S. H. (2017). Personal values in human life. Nature Human Behaviour, 1(9), 630-639. doi: 10.1038/s41562-01 7-0185-3

Schwartz, S. H. (1992). Universals in the content and structure of values: Theoretical advances and empirical tests in 20 countries. Advances in experimental social psychology, 25, 1-65. doi: 10.1016/S0065-2601(08) 60281-6

Schwartz, S. H. (1994). Are there universal aspects in the structure and contents of human values? Journal of social issues, 50(4), 19-45. doi: 10.1111/j.1540-45 60.1994.tb01196.x

Schwartz, S. H. (2003). A proposal for measuring value orientations across nations. Questionnaire Package of the European Social Survey, 259-290.

Schwartz, S. H. (2005). Basic human values: Their content and structure across countries. Valores $e$ comportamento nas organizações, 21-55.

Steinmetz, H., Isidor, R., \& Baeuerle, N. (2012). Testing the circular structure of human values: A metaanalytical structural equation modelling approach. Survey Research Methods, 6(1), 61-75. doi: 10.181 48/srm/2012.v6i1.5096

Torres, A. R. R., Pereira, C., Guimarães, J., Albernaz, M., Vieira, S., \& Barros, T. (2001). Valores e psicologia social: Modelos de análise. Estudos, 28(4), 539-559.

Vergara, A. I., \& Balluerka, N. (2000). Metodología en la investigación transcultural: perspectivas actuales. Psicothema, 12(2), 557-562. Recuperado de http:// www.psicothema.com/pdf/625.pdf

Wachelke, J., \& Bueno, L. (2015). Estudo exploratório das relações entre valores básicos pessoais e psicossociais. Avaliação Psicológica, 14(3), 353363. doi: 10.15689/ap.2015.1403.07

Zinbarg, R. E., Yovel, I., Revelle, W., \& McDonald, R. P. (2006). Estimating generalizability to a latent variable common to all of a scale's indicators: A comparison of estimators for $\omega_{\mathrm{h}}$. Applied Psychological Measurement, 30(2), 121-144. doi: 10.1177/0146 621605278814

Zubieta, E., Fernández, O., \& Sosa, F. (2012). Bienestar, valores y variables asociadas. Boletín de Psicología, 106, 7-27. Recuperado de https:/www. uv.es/seoane/ boletin/previos/N106-1.pdf 
Daniela Alonso

Instituto de Investigaciones Psicológicas, Facultad de Psicología, Universidad Nacional de Córdoba (UNC) y CONICET

Doctora en Psicología. Cuenta con investigaciones sobre participación política, ideología política, ciudadanía y legitimidad política democrática; incluyendo el análisis de dimensiones psicosociales y psicopolíticas involucradas en estos procesos.

ORCID: 0000-0003-2080-9571

Autor corresponsal: alonsodaniela.psi@gmail.com

Silvina Brussino

Instituto de Investigaciones Psicológicas, Facultad de Psicología, Universidad Nacional de Córdoba (UNC) y CONICET

Doctora en Psicología (Universidad Nacional de Córdoba), miembro de la Carrera de Investigador Científico y Tecnológico de CONICET y profesora de grado y posgrado. Dirige el equipo de investigación en psicología política (Facultad de Psicología, UNC) y tiene publicaciones sobre cultura política, participación política, ideología política, consumo de medios, entre otras; incluyendo el análisis de variables psicosociales y psicopolíticas involucradas en estos procesos. Cuenta además con publicaciones sobre métodos de investigación y estudios psicométricos.

ORCID: 0000-0002-1087-644X

sbrussino@conicet.gov.ar 